\title{
Del síntoma a la enfermedad: elevación de transaminasas
}

\author{
RB. Cortés Marina \\ Pediatra. CAP Montilivi-ICS. Girona. España.
}

Rev Pediatr Aten Primaria. 2009; I I (Supl I 7):s433-s436

Rosa Blanca Cortés Marina, rosapediatria@hotmail.com

\section{Resumen}

La transaminitis (elevación de AST-ALT) es un hallazgo habitual en la práctica clínica pediátrica. En ocasiones, su detección se produce en pacientes de cualquier edad sin síntomas de enfermedad hepática o biliar, tras una analítica solicitada por otro motivo.

Se presenta el caso clínico de una niña de 9 años, a la que se realiza una analítica para intentar llegar al diagnóstico etiológico de una reacción cutánea. Esta reacción apareció en el transcurso de un tratamiento antibiótico instaurado por una neumonía. Tras el hallazgo de hipertransaminemia y su posterior confirmación en analíticas posteriores, se aplica el protocolo de estudio y es diagnosticada de enfermedad de Wilson.

Palabras clave: Transaminasas, Hepatopatía, Enfermedad de Wilson.

Abstract

Increased transaminases (AST-ALT) is a common finding in paediatric clinical practice. Sometimes detection occurs in patients of any age with no symptoms of liver or biliary disease, foIlowing an analytical requested for another reason.

We report the case of a 9 year old girl, on whom analytical tests were performed in an attempt to establish the aetiology of a skin reaction. This reaction appeared during a course of an antibiotic treatment for pneumonia. After elevated transaminases and subsequent confirmation in subsequent analytical, applies the study protocol and is diagnosed with Wilson's disease.

Key words: Transaminases, Liver disease, Wilson's disease.

\section{Introducción}

Las transaminasas (ALT o GPT y AST o GOT) son enzimas intracelulares que catalizan reacciones de transaminación y que están localizadas en diversos tejidos del organismo, siendo la más específica de lesión hepática la GPT. La elevación anormal viene definida por valores superiores al rango de normalidad, que habitualmente se considera de 30-40 U/L. Su

La autora declara no presentar posibles conflictos de intereses en relación con la preparación y publicación de este artículo. 
aumento en sangre se debe a una destrucción celular o un trastorno de permeabilidad de la membrana de las células que las contienen ${ }^{1,2}$. No existe una clara correlación entre las cifras de transaminasas y el grado de lesión hepática. Pero sí podemos decir que por debajo de 3 años, generalmente, la hipertransaminemia es debida a una participación hepática en un proceso no hepático, siendo en niños mayores donde es más probable encontrar como causa de dicha elevación una hepatopatía de base.

\section{Caso clínico}

Niña de 9 años, sin antecedentes patológicos de interés, que acude por presentar exantema pruriginoso, con lesiones pápulo-eritematosas de pequeño tamaño que en algunas zonas se hacen confluyentes, afectando palmas y plantas. No existía enantema y la paciente se encontraba afebril. Como antecedente refiere estar tomando amoxicilina desde hace 9 días por una neumonía en LII diagnosticada en el hospital de referencia.

Como diagnóstico diferencial se plantea inicialmente con: infección por Micoplasma pneumoniae, exantema vírico (probable mononucleosis infecciosa), alergia medicamentosa a amoxicilina y toxicodermia.
Ante el cuadro clínico de la paciente se decide suspender el tratamiento antibiótico, se le pauta un antihistamínico para calmar el prurito y se le solicita una analítica de sangre.

Los resultados de la primera analítica fueron: VSG 31; $\mathrm{Hb} 14.1 \mathrm{~g} / \mathrm{dl}_{;} \mathrm{Ht}^{\circ} 41$; leucocitos 8.200 ( $N$ 44,7\%; L 42,1\%; M 9,3\%; E 3,6\%); plaquetas 564.000; GOT 91 U/L; GPT 220 U/L; ferritina 445; IgE 25,13 U/L; RAST a amoxicilina negativo; serología a Micoplasma pneumoniae IgG e IgM negativa.

Tras esta primera analítica, toma más fuerza la posible etiología vírica, probablemente un síndrome mononucleósico, y a las 2 semanas se realiza una analítica de control con los siguientes resultados: GOT 130 U/L; GPT 363 U/L; GGT 310 U/L; fosfatasa alcalina $352 \mathrm{U} / \mathrm{L}$; VSG 8; ferritina 138; Paul Bunnell negativo; serología hepatitis $A$, B y $C$ negativas; serología toxoplasma IgG e IgM negativa; serología CMV IgG positiva e IgM negativa; serología VEB IgG positiva e IgM negativa.

Llegados a este punto se decide repetir la analítica al mes ampliando el estudio y descartando las causas más frecuentes de transaminitis. Los resultados fueron: GOT 228 U/L; GPT 483U/L; GGT 257U/L; CPK 87 U/L; alfa-1-antripsina $147 \mathrm{mg} / \mathrm{dl}$; ceruloplasmina $3,23 \mathrm{mg} / \mathrm{dl}$ (22-58); cobre en plasma 43 mcg/dl (80- 
160); anticuerpos antinucleares, antiDNA, anti-LKM negativos; IgA antitransglutaminasa negativos; alfa-fetoproteína negativa; serología brucela negativa, serología parvovirus B19 negativa.

Tras esta analítica la sospecha se centra en la enfermedad de Wilson. Para completar el estudio se solicita cobre en orina, siendo el resultado de $170 \mathrm{mcg} / 24 \mathrm{~h}$ (normal: 0-60) y ecografía hepática donde se visualiza leve esteatosis.

Con la sospecha de enfermedad de Wilson, se envía a la unidad de hepatología pediátrica de hospital de referencia donde confirman el diagnóstico e inician tratamiento con D-penicilamina.

\section{Discusión}

Algunos autores hablan que entre un 1-4\% de la población asintomática puede presentar elevación sérica de las transaminasas. En general, cualquier daño en el hígado producirá una elevación de estas enzimas y el diagnóstico etiológico requerirá un análisis exhaustivo de la historia clínica, la exploración física y pruebas complementarias de laboratorio e imagen. En ocasiones, la prueba definitiva puede llegar a ser la biopsia hepática ${ }^{1,3}$.

Si se confirma la elevación, dentro de una primera fase de estudio se debe realizar los siguientes estudios ${ }^{3-5}$ : hemo- grama y VSG, urea y creatinina, bioquímica con glucemia y perfil lipídico, estudio de coagulación, perfil hepático, bilirrubina, fosfatasa alcalina, serología hepatitis $A, B, C$, virus de Epstein-Barr y citomegalovirus, proteínas totales, enzimas musculares, y orina y sedimento.

Si persiste la hipertransaminemia los estudios que se deben realizar en una segunda fase son: TSH, estudio del metabolismo del hierro, ceruloplasmina sérica, cupremia, anticuerpos antinucleares (ANA), anticuerpos antimitocondriales (AMA), anticuerpos antimúsculo liso (SMA), anticuerpos microsomales antihígado y riñón (anti-LKM), anticuerpos antiproteína soluble hepática (anti-LSP), anticuerpos antimembrana hepática (anti-LMA), alfa1-antitripsina, inmunoglobulinas, anticuerpos igA antitransglutaminasa, proteinograma, test del sudor, cuerpos reductores en orina y estudio para descartar enfermedades metabólicas con afectación hepática, marcadores tumorales si la historia clínica lo sugiere y ecografía abdominal, otras pruebas de imagen si es preciso y en los casos que se requiera, una biopsia hepática.

La enfermedad de Wilson, también conocida como degeneración hepato-lenticular, es una enfermedad congénita, con un patrón de herencia autosómica recesiva, caracterizada por la acumulación de 
grandes cantidades de cobre en hígado y cerebro y que presenta un desenlace fatal sin tratamiento. La enfermedad es debida a un déficit en la excreción biliar de este metal. El perfil bioquímico que encontramos en la mayor parte de los casos corresponde: a niveles bajos de ceruloplasmina sérica, cupremia baja y excreción urinaria de cobre aumentada.

El tratamiento tiene como principal objeto la eliminación del cobre acumulado en el organismo, siendo el fármaco más activo la D-penicilamina. Con el tratamiento adecuado los pacientes afectos que estén asintomáticos nunca desarro- llarán síntomas y la mayor parte de los sintomáticos experimentan una mejoría o la resolución de los mismos ${ }^{5-7}$.

\section{Conclusiones}

Es necesario abordar de forma estructurada y multidisciplinaria el estudio de la hipertransaminemia en los pacientes pediátricos.

Hay que mantener los controles hasta la identificación de la causa o su total normalización y ante una elevación persistente de las transaminasas, siempre pensar en una posible causa muscular o metabólica 4 .

\section{Bibliografía}

1. Sánchez Milla JJ, Fernández Álvarez FJ. Valoración inicial de las hipertransaminasemias en los reconocimientos médicos. Algoritmo diagnóstico. SESLAP. 2004;1(10).

2. Romero Frais MJ. Guía clínica. A Coruña: SERGAS; 2001.

3. Díaz Otero A. Guías clínicas Fisterra 2009. Transaminasas [consultado el 10/09/2009]. Disponible en www.fisterra.com/guias2/transami nasas.asp
4. Díez R. Elevació asimptomàtica de les transaminases en l'infant. Pediatr Cat. 2008;68:16871.

5. Richard E, Behrman MD. Tratado de Pediatría de Nelson. 17. a ed. Elsevier; 2004. p. 1312 21.

6. Bruguera M. Enfermedad de Wilson. Gastroenterol Hepatol. 2006;29(1):29-33.

7. Solís Muñoz P, Solís Herruzo JA. Enfermedad de Wilson. Una enfermedad rara pero presente. Rev Esp Enferm Dig. 2008;100(8). 